\title{
Managing complications of pleural procedures
}

\author{
John G. Williams ${ }^{1}$, Andrew D. Lerner ${ }^{2}$ \\ ${ }^{1}$ Division of Pulmonary and Critical Care Medicine, Department of Medicine, University of Maryland School of Medicine, Baltimore, MD, USA; \\ ${ }^{2}$ Department of Pulmonary and Critical Care Medicine, Johns Hopkins University School of Medicine, Baltimore, MD, USA \\ Contributions: (I) Conception and design: Both authors; (II) Administrative support: Both authors; (III) Provision of study materials or patients: \\ Both authors; (IV) Collection and assembly of data: Both authors; (V) Data analysis and interpretation: Both authors; (VI) Manuscript writing: Both \\ authors; (VII) Final approval of manuscript: Both authors. \\ Correspondence to: Dr. Andrew D. Lerner, MD. 5215 Loughboro Road NW, Suite 420 Washington, DC 20016, USA. Email: alerne11@jhmi.edu.
}

\begin{abstract}
Pleural disease is common and often requires procedural intervention. Given this prevalence, pleural procedures are performed by a wide range of providers with varying skill level in both medical and surgical specialties. Even though the overall complication rate of pleural procedures is low, the proximity to vital organs and blood vessels can lead to serious complications which if left unrecognized can be life threatening. As a result, it is of the utmost importance for the provider to have a firm grasp of the local anatomy both conceptually when preparing for the procedure and physically, via physical exam and the use of a real time imaging modality such as ultrasound, when performing the procedure. With this in mind, anyone who wishes to safely perform pleural procedures should be able to appropriately anticipate, quickly identify, and efficiently manage any potential complication including not only those seen with many procedures such as pain, bleeding, and infection but also those specific to procedures performed in the thorax such as pneumothorax, re-expansional pulmonary edema, and regional organ injury. In this article, we will review the basic approach to most pleural procedures along with essential local anatomy most often encountered during these procedures. This will lay the foundation for the remainder of the article where we will discuss clinical manifestations and management of various pleural procedure complications.
\end{abstract}

Keywords: Pleural procedure; pneumothorax; complications; management

Submitted Jan 18, 2020. Accepted for publication Apr 29, 2020.

doi: 10.21037/jtd-2019-ipicu-04

View this article at: http://dx.doi.org/10.21037/jtd-2019-ipicu-04

\section{Introduction}

Pleural procedures are a cornerstone for the practicing physician. Through a relatively narrow skill set, one can provide a wide array of services from invaluable diagnostic information to long term therapeutic intervention. In addition to breadth of utility, pleural procedures are likely popular due to their attractive risk to benefit ratio. Overall, the complication rate of most non-surgical pleural procedures is low. However, given the proximity to vital organs, complications can occur and the inability to recognize and appropriately manage these complications could prove fatal. In this article we review identification and management of complications of common pleural procedures.

\section{General approaches and minimizing risk}

Diagnostic and therapeutic pleural procedures come in a variety of forms. They include pleural fluid drainage (via needle aspiration, thoracentesis, tube thoracostomy, or indwelling pleural catheter placement), pleural biopsy (via medical thoracoscopy or closed pleural biopsy), and instillation of therapeutic agents (such as chemical pleurodesis). Many of these procedures share a common methodology whereby local anesthesia is applied and pleural access is gained via transcutaneous needle insertion or blunt dissection. Complications can occur both intra- and postprocedure and often revolve around the underlying anatomy and patient comorbidities.

As such, a comprehensive understanding of the 

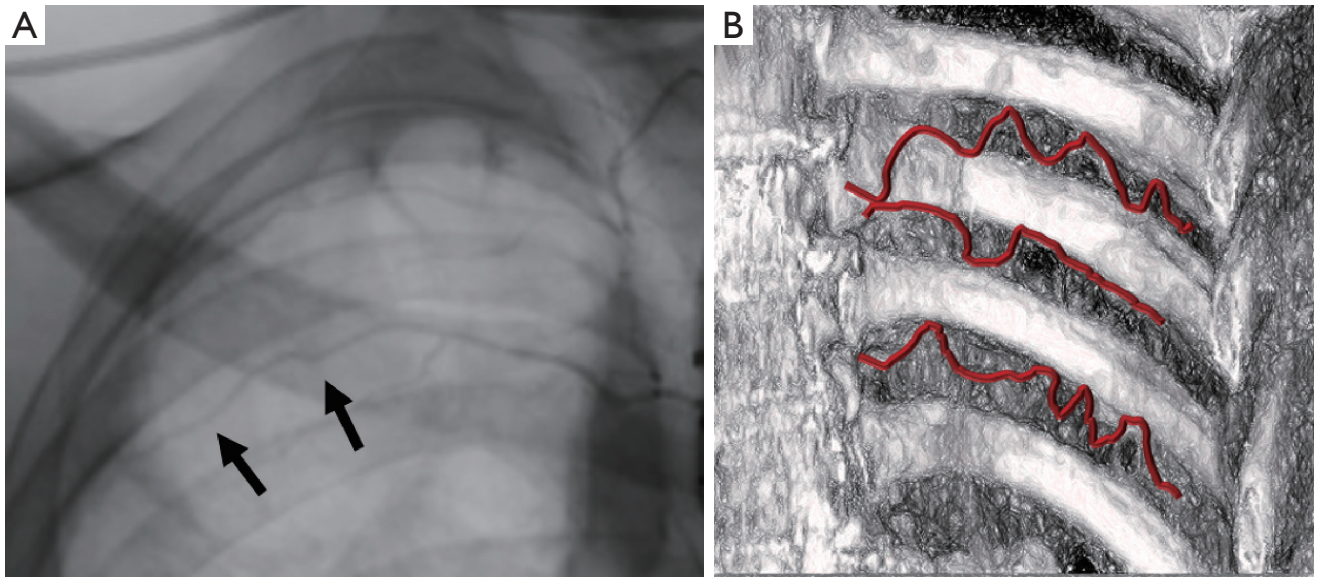

Figure 1 Intercostal artery (ICA) course as seen on (A) angiogram showing a mild variation (black arrows) in a young man and (B) a digitally enhanced CT reconstruction highlighting the tortuous course of an ICA in an elderly man (red lines).

underlying thoracic anatomy is critical in minimizing risk of complication. The major relevant structures include the chest wall, ribs, intercostal neurovascular bundle (consisting of an intercostal vein, artery, and nerve), heart, lungs, diaphragm, and subdiaphragmatic organs. The intercostal neurovascular bundle tracks along the inferior margin of the associated rib and injury to this bundle can increase the risk of bleeding and pain. In light of this anatomical relationship, access to the pleural space is typically approached over the superior rib margin. It should be noted that this vesselto-rib relationship is less reliable along the posterior and medial aspect of the ribs $(1,2)$, particularly in elderly, and therefore a lateral approach is generally preferred to lessen risk of injury (Figure 1).

The adoption of procedural ultrasound has further helped reduce the rate of complications when accessing the pleural space. Ultrasound is used to help locate an appropriate puncture site by identifying the pleural space and surrounding structures such as the heart, lungs, diaphragm, liver, and spleen. In one study comparing ultrasound to physical exam in selecting the appropriate puncture site, ultrasound prevented organ puncture in $10 \%$ of overall cases and increased the accuracy of the puncture site by $26 \%$ (3). Guidelines now recommend the routine use of thoracic ultrasound guidance for most pleural procedures (4).

Proper patient positioning is also an important aspect of many pleural procedures and can help reduce the risk of complications. Three commonly used and well accepted positions expose the provider to the 'triangle of safety,' which is the preferred area of entry when not using ultrasound localization or when not limited by loculated effusion or apical pneumothorax (4). The 'triangle of safety' is bordered by the superior aspect of the fifth rib inferiorly, the lateral edge of latissimus dorsi posteriorly, and the lateral edge of pectoralis major anteriorly (Figure 2). This triangle is appropriately exposed when the patient is lying in the lateral decubitus position, sitting upright and leaning forward with arms raised, and lying in the semi-recumbent position with the arm raised above the head.

\section{Identification and management of pleural complications}

\section{Pneumothorax}

Iatrogenic pneumothorax can occur with all pleural procedures and at varying rates. Most commonly reported as a complication of pleural fluid aspiration, iatrogenic pneumothorax has been found in $0.3-1.5 \%$ of cases using ultrasound guidance and $5.7-15 \%$ without ultrasound (4-6). Physiologically, pneumothoraces develop via different mechanisms. First, and most concerning, is by direct injury to the visceral pleura via laceration or puncture. Another mechanism is through the entrainment of outside air into the pleural space via the needle, catheter, or incision site. Finally, pneumothorax ex vacuo can occur when fluid is removed in the setting of non-expandable lung, leaving a negative pressure space. However, pneumothorax ex vacuo is typically a benign condition that is not universally considered a complication but rather a physiologic sequalae of non-expandable lung and does not likely benefit from chest tube insertion (7). Distinguishing between the types 


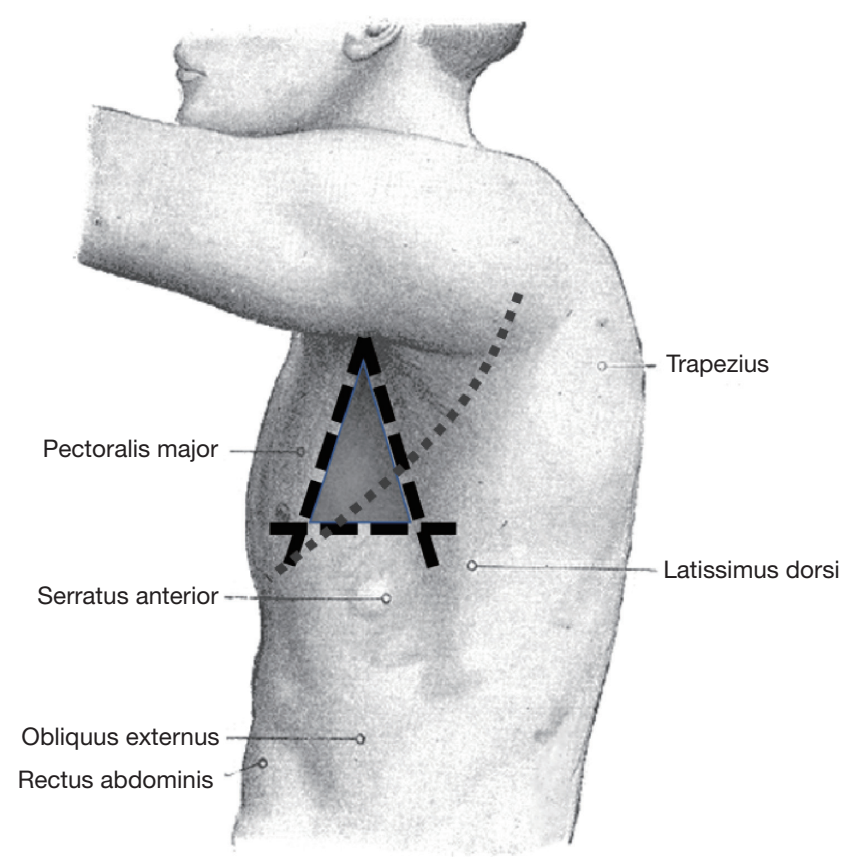

Figure 2 Triangle of safety as outlined by the superior aspect of the fifth rib inferiorly, lateral edge of latissimus dorsi posteriorly, and lateral edge of pectoralis major anteriorly. Notably, the left oblique fissure (small dashed line) passes through the triangle of safety, which leads to the risk of chest tube placement within the fissure. Original image by Henry Vandyke Carter/Public Domain and modified by the authors.

of pneumothorax based solely on chest radiograph can be challenging. One pearl to consider is that pneumothorax ex vacuo often appears as ipsilateral hemi-thoracic volume loss (elevated hemidiaphragm, narrowed rib spaces, and mediastinal shift toward the side of the procedure) (Figure $3 A$ ) while pneumothorax due to pulmonary injury can appear more similarly to spontaneous pneumothorax (deep sulcus, widened rib spaces, and mediastinal shift away from the procedural side) (Figure $3 B)(7,8)$.

Chest radiograph is most commonly used to assess for post-procedural pneumothorax. However, some evidence suggests that routine chest radiography is not indicated in simple thoracentesis and should only be obtained if air is aspirated during the procedure, multiple attempts were made, or the patient develops shortness of breath, hypoxia, or hemodynamic instability (4,9-11). Alternatively, thoracic ultrasound can be used to assess for lung sliding in ruling "out" a pneumothorax at the site of the ultrasound probe (12). However, since lack of lung sliding can also be seen in certain conditions of expanded lung (i.e., pleurodesed lung), ultrasound is limited in its ability to rule "in" a pneumothorax. Nevertheless, the "lung point sign" under ultrasound, where sliding lung is seen immediately next to air, is a confirmatory finding for pneumothorax (12).

The management of post-procedural pneumothorax is often determined by the size of the pleural air space as well as the development of clinical symptoms. Most iatrogenic pneumothoraces are small and can be observed clinically without decompression (8). Regarding size, some guidelines recommend the following regarding postprocedural pneumothorax: (I) if the pneumothorax is small $(<2 \mathrm{~cm}$ between the chest wall and lung at the apex) and the patient is asymptomatic observation and supplemental oxygen are all that is needed. (II) If the patient is symptomatic, the pneumothorax is large ( $>2 \mathrm{~cm}$ between chest wall and lung at the apex), or if the pneumothorax is expanding, tube thoracostomy is indicated (8). In these instances, small bore $(\leq 14 \mathrm{Fr}$ ) chest tubes placed using the Seldinger technique have been shown to be as effective and less painful than large bore chest tubes and, as a result, are recommended as first line therapy $(4,8)$. Large-bore chest tubes, placed using blunt dissection, can be used for large volume air leaks when small bore drainage is insufficient or unavailable.

Treatment via high supplemental fraction of inspired oxygen functions to reduce the total pressure of gases in the pleural capillaries by reducing the partial pressure of nitrogen (13). This creates an increase in the pressure gradient between the pleural capillaries and pleural space which facilitates absorption of air from the pleural cavity.

Though rare, a persistent air leak (PAL) can occur following pleural procedures. This is identified by the presence of persistent air bubbles in the water seal chamber of the chest tube drainage system lasting at least 3-7 days and can be representative of a bronchopleural or alveolarpleural fistula. Treatment can range from prolonged pleural drainage (with or without an ambulatory Heimlich valve) to pleurodesis. There remains a lack of evidence-based data to support the best therapeutic approach. However, unlike post-surgical PAL, pleurodesis is often not needed for iatrogenic pleural cases since these typically resolve with watchful waiting alone (14).

Ultimately, management of post-procedural pneumothoraces revolve around early recognition followed by either clinical observation or tube thoracostomy. Of note, pneumothoraxex-vacuo is a benign condition and rarely requires decompression. 


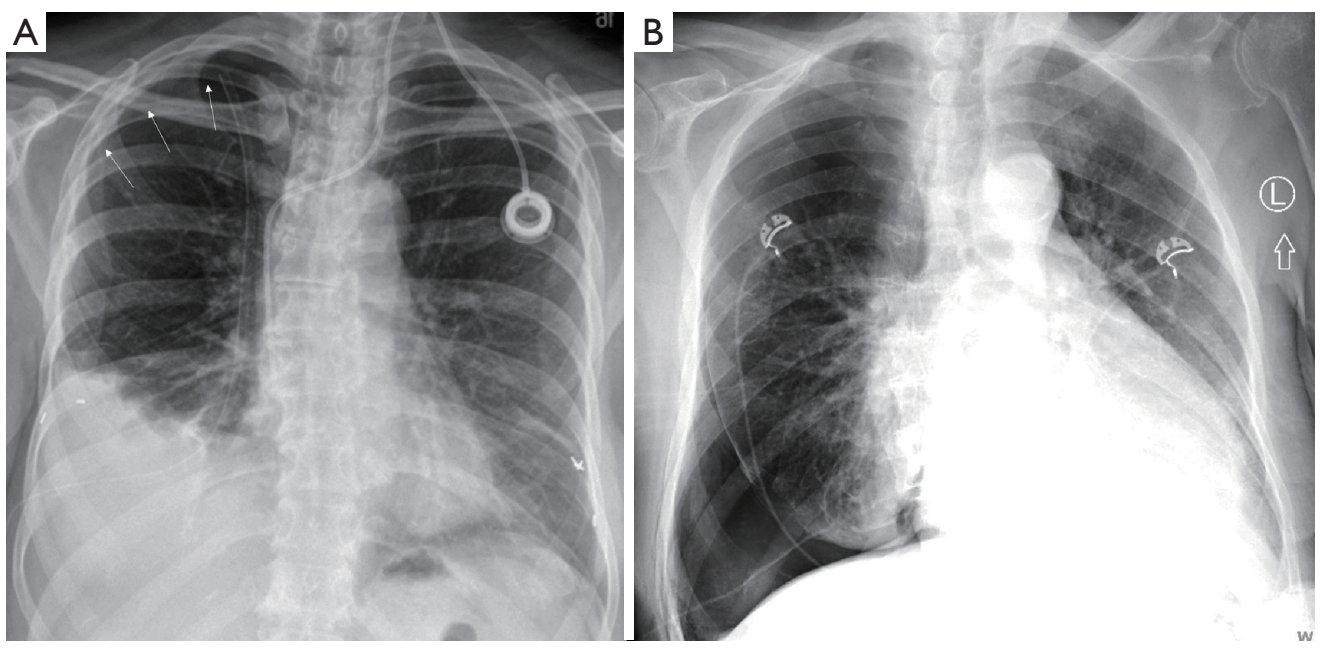

Figure 3 Features of pneumothorax comparing anteroposterior chest X-rays of (A) pneumothorax ex vacuo (arrows) characterized by ipsilateral volume loss and (B) typical pneumothorax with a deep sulcus sign.

\section{Re-expansion pulmonary edema (RPE)}

$\mathrm{RPE}$ is thought to occur as a result of rapid large volume removal or profoundly negative pleural pressure during pleural aspiration $(15,16)$. It is traditionally considered to be a rare but potentially life-threatening complication of therapeutic pleural aspiration. The true incidence of RPE is unknown with small case series and reviews reporting an incidence between $0.2 \%$ and $14 \%$ (17). The majority of those cases appear to be isolated radiographic findings in asymptomatic patients and clinically symptomatic RPE is reported to occur in $<1 \%$ of cases (17).

$\mathrm{RPE}$ is suspected when a patient develops dyspnea, cough, and hypoxia in the minutes to hours following pleural aspiration. However, it can occur anytime within the first 24 hours (16). Radiographically, RPE can present as ipsilateral and less frequently as bilateral ground glass opacities on chest imaging (Figures 4,5) (18).

Once RPE occurs, treatment is supportive and based on clinical severity, which may involve supplemental oxygen, non-invasive positive pressure, or mechanical ventilation $(15,16)$. The use of medications such as steroids and diuretics during treatment has not been proven to be beneficial and remains uncertain (19). Close cardiopulmonary monitoring in symptomatic patients will help identify the need for escalation of supportive care. RPE is typically self-limited following supportive measures (20).

Prevention of RPE has been proposed by various means. Prior studies have suggested that risk of RPE is dependent on the volume of fluid removed and the degree of negative pressure developed within the pleural space (17). From this, expert opinion has historically recommended terminating pleural fluid drainage at 1 or $1.5 \mathrm{~L}$ if pleural pressures are not being monitored $(15,21,22)$. However, this arbitrary cutoff point has been challenged and additional data suggest that RPE is independent of the volume of fluid removed and absolute change in pleural pressure or elastance (17). As such, these studies recommended complete drainage as long as chest discomfort or end-expiratory pleural pressure less than $-20 \mathrm{cmH}_{2} \mathrm{O}$ does not develop (17).

Monitoring of pleural pressures via manometry can be considered during large volume thoracenteses or in cases where non-expandable lung is suspected (17). However, in a prospective trial on the use of pleural pressure manometry for large volume thoracentesis, manometry did not alter the primary outcome of procedure related chest discomfort (23).

\section{Pain}

Pain is a common complication that may occur in all forms of pleural procedures. Pain varies depending on the type of procedure and underlying indication. Pain is reported following thoracentesis in anywhere from $5-39 \%$ of patients $(5,6,24)$. In an audit by Hooper et al., $8 \%$ reported pain during chest tube placement and $15.6 \%$ reported delayed pain after chest tube placement (5). Patients also had more pain when a chest tube was placed for pneumothorax (5).

One classic syndrome is re-expansional pain in the setting of pleural drainage with non-expandable lung. 

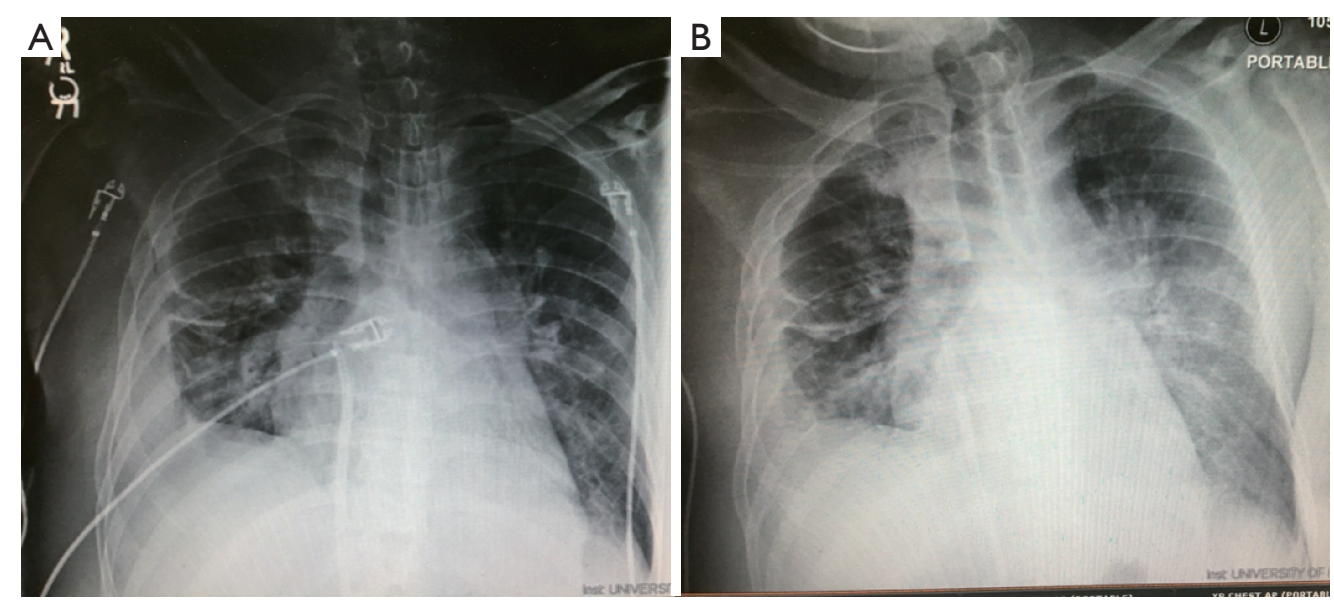

Figure 4 Features of RPE in a 58-year-old man with systolic heart failure and end-stage renal disease who underwent ultrasound guided thoracentesis and removal of approximately $1 \mathrm{~L}$ of straw colored fluid. (A) Chest X-ray immediately post left-sided thoracentesis demonstrates a clear left lung field. (B) One hour post-thoracentesis he developed worsening hypoxemia and repeat chest X-ray demonstrates left sided air-space opacities characteristic of RPE. RPE, re-expansion pulmonary edema.
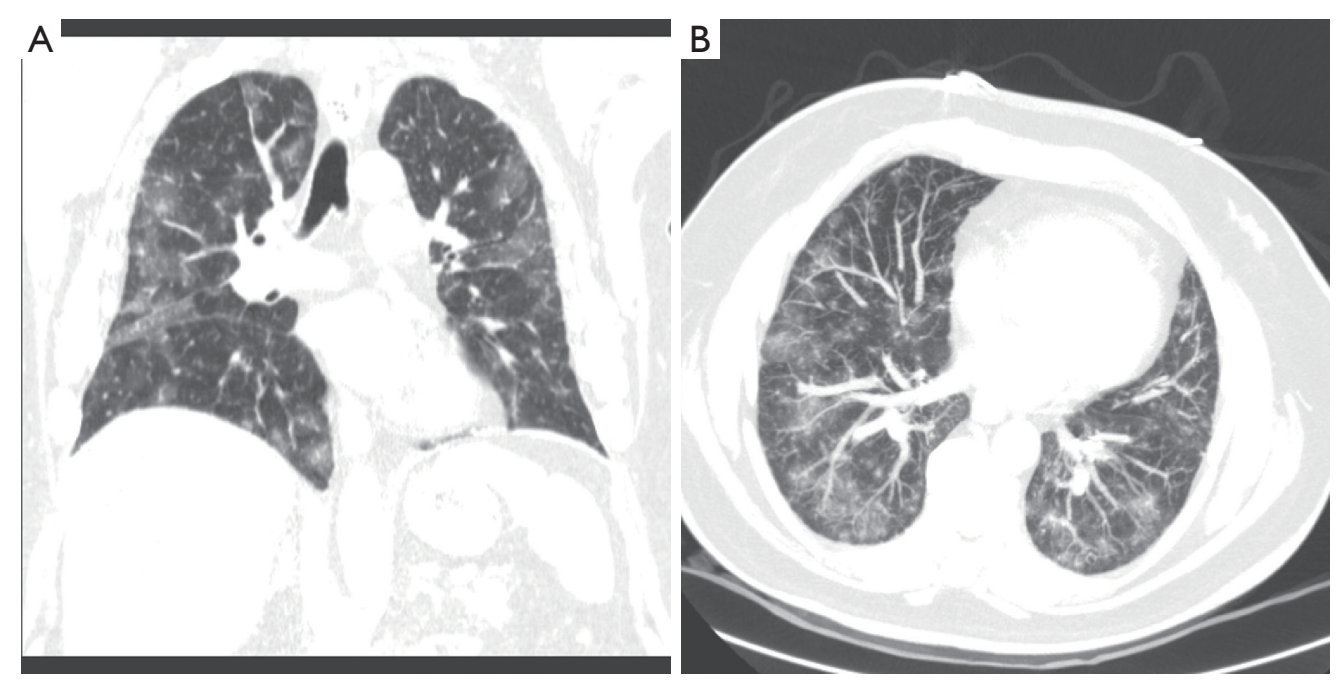

Figure 5 Bilateral re-expansional pulmonary edema following thoracentesis as seen on (A) coronal and (B) axial cuts of a chest computed tomography scan.

This can be minimized by stopping the pleural drainage procedure if patient experiences discomfort.

Pain is also a common complication of chemical pleurodesis. Patients undergoing pleurodesis should be counseled on the risk of pain and given adequate analgesia in the post-operative setting. Many studies have compared pain scores between agents. However, when compared in meta-analysis, no difference was found between them (25).

Although data is limited on the ideal pain control regimen following pleurodesis, nonsteroidal antiinflammatory drugs have been found to provide similar pain relief as opiates without increasing the risk of pleurodesis failure (26).

\section{Bleeding}

The risk of significant bleeding from pleural procedures is low. Bleeding may be arterial or venous and is often related 

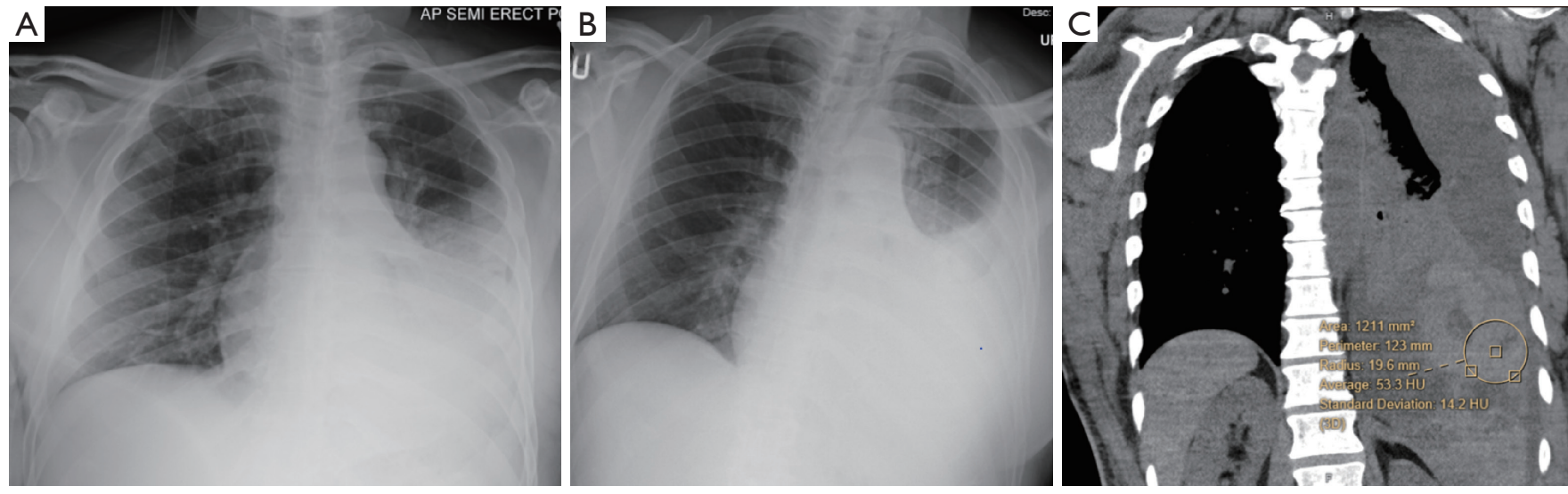

Figure 6 Hemothorax following thoracentesis. A 52-year-old patient with parapneumonic effusion underwent thoracentesis using an 8 French needle-catheter. The patient was started on therapeutic anticoagulation 24 hours post-procedure and developed hemorrhagic shock approximately 36 hours post-procedure. (A) Chest X-ray immediately following left sided thoracentesis. (B) Chest X-ray 36 hours after thoracentesis showing re-accumulation of fluid in the left pleural space. (C) Chest computed tomography scan with Hounsfield units consistent with hemothorax. The patient underwent video assisted thoracoscopic surgery that identified a bleeding intercostal vessel that was subsequently cauterized with Argon plasma coagulation.

to damage to the intercostal vessels. Bleeding is suspected in the post-procedure setting with the rapidly reaccumulating pleural fluid, which can be visualized sonographically or radiographically, or with the rapid onset of respiratory symptoms such as shortness of breath or chest pain.

The first step in prevention of this complication is the avoidance of elective procedures while patients are receiving therapeutic anticoagulation. Notably, aspirin is believed to be safe during pleural procedures $(27,28)$. For urgent procedures, or if anticoagulation cannot be stopped, the risk-benefit ratio needs to be assessed and discussed with the patient.

The majority of post-procedural bleeding is superficial and can be managed with external pressure. However, massive hemorrhage can occur (Figure 6). Once identified, the first goal is simultaneous resuscitation and cessation of any ongoing bleeding. Resuscitation will be patient and resource specific with blood product transfusion being the ideal fluid in hemodynamic instability. Cessation of ongoing bleeding starts with directed external pressure and may progress to surgical intervention. This typically includes urgent consultation to thoracic surgery for consideration of thoracotomy and to interventional radiology for consideration of transcatheter arterial embolization (Figure 7). The choice of intervention is patient and hospital specific where, when available, the minimally invasive approach for transcatheter arterial embolization may be an option for patients otherwise not appropriate for surgery $(29,30)$. Nevertheless, the initial treatment of iatrogenic hemothorax often includes the placement of a chest tube. The size of the chest tube (large bore $v s$. small bore) is still in debate with the general goal to adequately drain the blood while taking into consideration the risks of tube clotting with smaller tubes and the degree of patient discomfort with larger tubes (31-33).

\section{Injury to the liver, spleen, and diaphragm}

With the advent of point of care ultrasound, injury to the liver, spleen, and diaphragm has significantly decreased (3). Nevertheless, injury is still possible and has been reported to occur in $0.3 \%$ of thoracentesis and $0.6 \%$ of chest tube placement (Figures 8,9) (5). When using ultrasound, definitive identification of the diaphragm and appropriate cranial, caudal alignment are will help iatrogenic complications. Organ injury remains a consideration in the setting of any post-procedural clinical decline. In the setting of injury to surrounding structures, urgent surgical referral may be warranted.

\section{Infection}

Infection at the site of pleural procedures is rare when performed under sterile conditions (5). Indwelling tunneled 

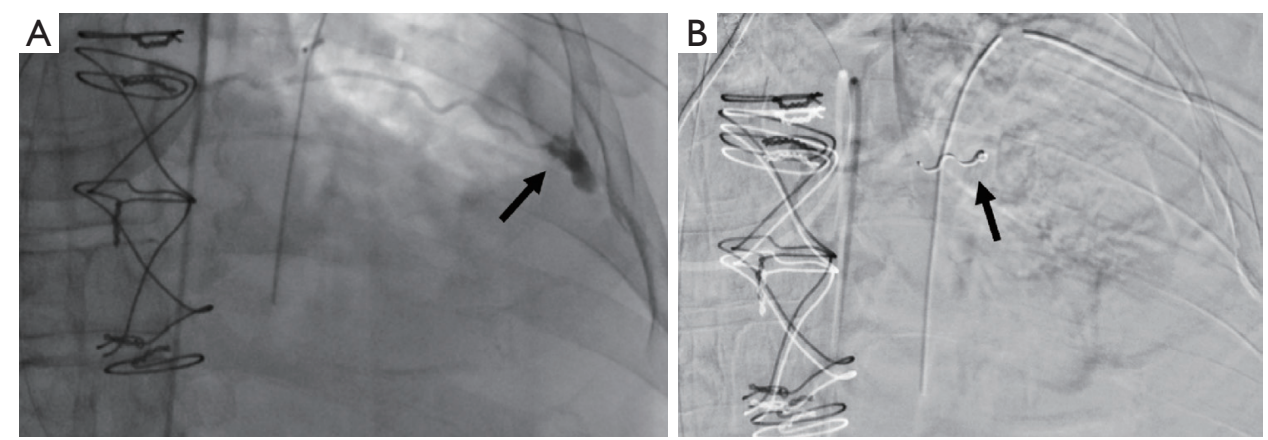

Figure 7 ICA angiogram demonstrating (A) tortuous path of the ICA along with the site of active bleeding (arrow) in patient who developed hemorrhagic shock 10 hours post ultrasound guided left thoracentesis. (B) The ICA was successfully embolized (arrow) and no flow was demonstrated across the injury site. ICA, intercostal artery.
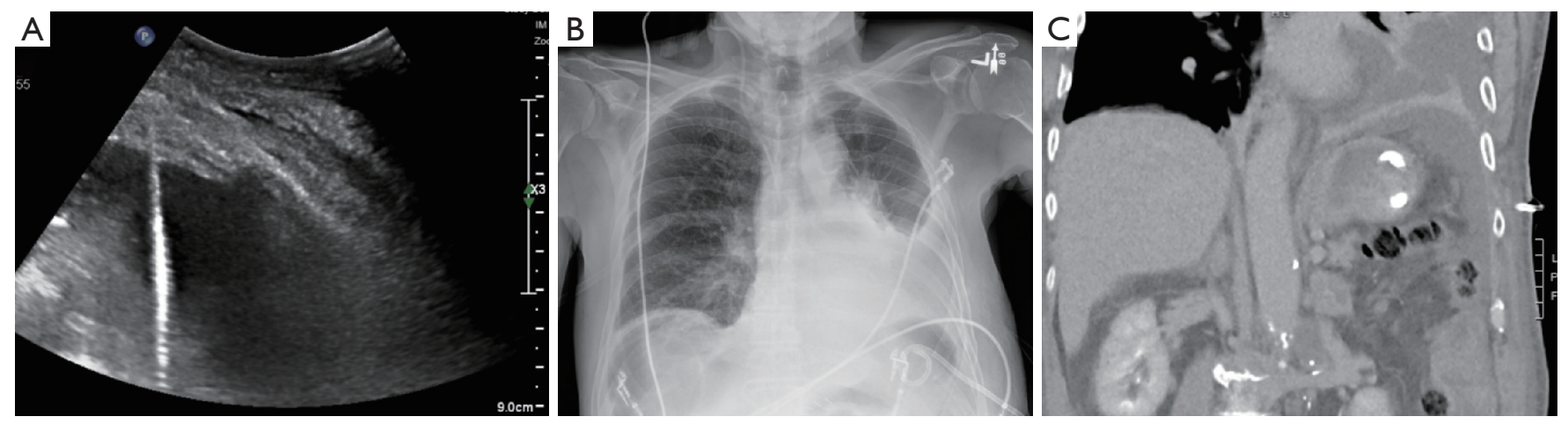

Figure 8 Small-bore catheter placed in the stomach. A 70-year-old man underwent ultrasound guided chest tube placement for parapneumonic effusion. (A) Intraoperative ultrasound image showing guidewire. (B) Chest X-ray showing sub-diaphragmatic small-bore catheter placement. (C) Chest computed tomography scan confirming placement of the small-bore catheter in the stomach. Immediately following catheter placement, the tube drained $300 \mathrm{~mL}$ of green, bilious fluid. The patient then underwent exploratory laparotomy with removal of the intragastric small-bore catheter, gastrotomy repair, and placement of gastro-jejunal tube and left small-bore chest tube.
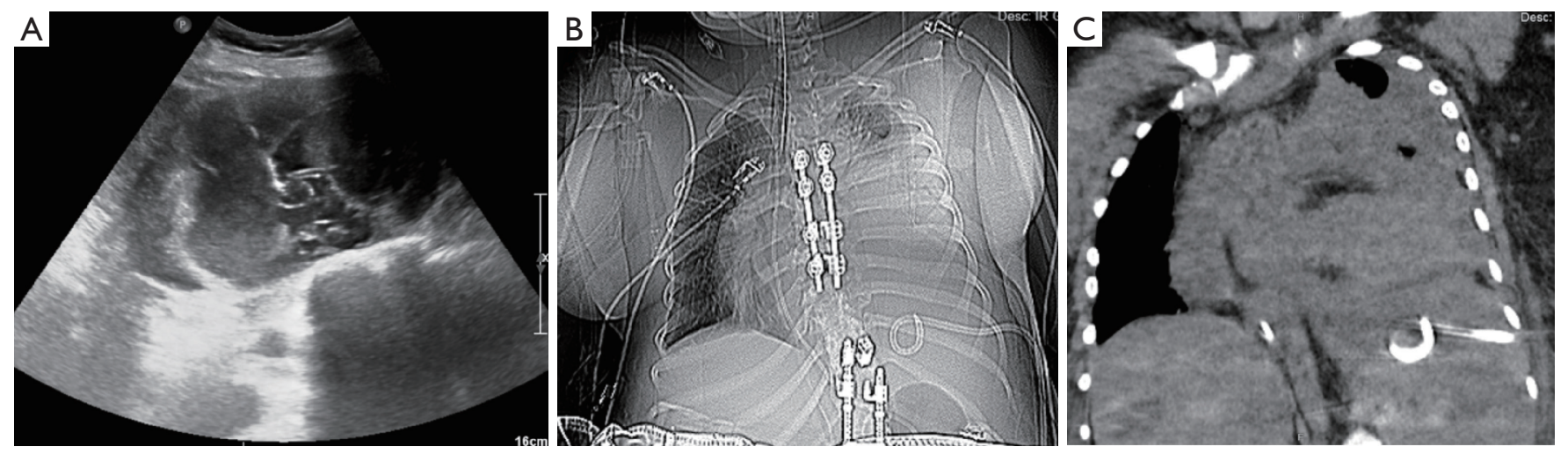

Figure 9 Small-bore catheter traversing the spleen. A 32-year-old woman who underwent ultrasound guided small-bore catheter placement for left sided empyema. (A) Intraoperative ultrasound image showing guidewire. (B) Scout film from CT showing sub-diaphragmatic smallbore catheter placement. (C) Chest CT scan confirming subdiaphragmatic small-bore catheter placement. The patient required packed red blood cell transfusion and underwent successful catheter removal. A subsequent small-bore catheter was placed for the left sided empyema. CT, computed tomography. 
pleural catheters are often placed for recurrent malignant effusions and can remain in place for months. These patients can be immunosuppressed and at increased risk of infection. With proper care of indwelling catheters, the rate of infection is low (34). Once identified, infections should be managed with appropriate antibiotics. Removal of any indwelling devices is often based on the extent and severity of the infection. Mild superficial cellulitis often can be treated with antibiotics without catheter removal $(34,35)$. Additionally, pleural infections short of frank empyema can usually be managed with antibiotics and the instillation of fibrinolytics agents, which have been shown to reduce the duration of infection and need for surgery $(36,37)$. Frank empyema not responding to antibiotics therapy necessitates indwelling catheter removal and potential surgical referral $(34,35,37)$.

\section{Conclusions}

Pleural procedures are commonplace in modern medical practice and, while they are relatively safe, complications can be severe and prove fatal. With this in mind, one should approach any pleural procedure with the appropriate knowledge and training in order to anticipate, identify, and manage the potential complications that may arise.

\section{Acknowledgments}

Funding: None.

\section{Footnote}

Provenance and Peer Review: This article was commissioned by the Guest Editors (Jonathan S. Kurman, Ashutosh Sachdeva and Rahul Nanchal) for the series "Interventional Pulmonology in the Intensive Care Unit Environment" published in fournal of Thoracic Disease. The article has undergone external peer review.

Conflicts of Interest: Both authors have completed the ICMJE uniform disclosure form (available at http://dx.doi. org/10.21037/jtd-2019-ipicu-04). The series "Interventional Pulmonology in the Intensive Care Unit Environment" was commissioned by the editorial office without any funding sponsorship. The authors have no other conflicts of interest to declare.

Ethical Statement: The authors are accountable for all aspects of the work in ensuring that questions related to the accuracy or integrity of any part of the work are appropriately investigated and resolved.

Open Access Statement: This is an Open Access article distributed in accordance with the Creative Commons Attribution-NonCommercial-NoDerivs 4.0 International License (CC BY-NC-ND 4.0), which permits the noncommercial replication and distribution of the article with the strict proviso that no changes or edits are made and the original work is properly cited (including links to both the formal publication through the relevant DOI and the license). See: https://creativecommons.org/licenses/by-nc-nd/4.0/.

\section{References}

1. Sachdeva A, Shepherd R, Lee H. Thoracentesis and Thoracic Ultrasound: State of the Art in 2013. Clin Chest Med 2013;34:1-9.

2. Helm EJ, Rahman NM, Talakoub O, et al. Course and variation of the intercostal artery by $\mathrm{CT}$ scan. Chest 2013;143:634-9.

3. Diacon AH, Brutsche $M H$, Solèr $M$. Accuracy of pleural puncture sites: A prospective comparison of clinical examination with ultrasound. Chest 2003;123:436-41.

4. Havelock T, Teoh R, Laws D, et al. Pleural procedures and thoracic ultrasound: British Thoracic Society pleural disease guideline 2010. Thorax 2010;65 Suppl 2:ii61-76.

5. Hooper CE, Welham SA, Maskell NA. Pleural procedures and patient safety: A national BTS audit of practice. Thorax 2015;70:189-91.

6. Seneff MG, Corwin RW, Gold LH, et al. Complications associated with thoracentesis. Chest 1986;90:97-100.

7. Ponrartana S, LaBerge J, Kerlan R, et al. Management of patients with "ex vacuo" pneumothorax after thoracentesis. Acad Radiol 2005;12:980-6.

8. MacDuff A, Arnold A, Harvey J. Management of spontaneous pneumothorax: British Thoracic Society Pleural Disease Guideline 2010. Thorax 2010;65 Suppl 2:ii18-31.

9. Doyle JJ, Hnatiuk OW, Torrington KG, et al. Necessity of routine chest roentgenography after thoracentesis. Ann Intern Med 1996;124:816-20.

10. Capizzi SA, Prakash UB. Chest roentgenography after outpatient thoracentesis. Mayo Clin Proc 1998;73:948-50.

11. Petersen WG, Zimmerman R. Limited utility of chest radiograph after thoracentesis. Chest 2000;117:1038-42.

12. Shostak E, Brylka D, Krepp J, et al. Bedside sonography 
for detection of postprocedure pneumothorax. J

Ultrasound Med 2013;32:1003-9.

13. Sahn SA, Heffner JE. Spontaneous pneumothorax. N Engl J Med 2000;342:868-74.

14. Lerner AD, Yarmus L, Gorden JA, et al. Intrabronchial valves for persistent air-leaks: what's the verdict? Expert Rev Respir Med 2016;10:1151-3.

15. Mahfood S, Hix W, Aaron B, et al. Reexpansion Pulmonary Edema. Ann Thorac Surg 1988;45:340-5.

16. Trachiotis GD, Vricella LA, Aaron BL, et al. Reexpansion pulmonary edema. Updated in 1997. Ann Thorac Surg 1997;63:1206-7.

17. Feller-Kopman D, Berkowitz D, Boiselle P, et al. LargeVolume Thoracentesis and the Risk of Reexpansion Pulmonary Edema. Ann Thorac Surg 2007;84:1656-61.

18. Gleeson T, Thiessen R, Müller N. Reexpansion pulmonary edema: computed tomography findings in 22 patients. J Thorac Imaging 2011;26:36-41.

19. Alqadi R, Fonseca-Valencia C, Viscusi M, et al. Reexpansion Pulmonary Edema Following Thoracentesis. R I Med J (2013) 2013;96:38-40.

20. Sherman SC. Reexpansion pulmonary edema: A case report and review of the current literature. J Emerg Med 2003;24:23-7.

21. Light RW. Pleural Diseases 4th ed. Baltimore: Lippincott, Williams and Wilkins; 2001.

22. Sautter RD, Dreher WH, MacIndoe JH, et al. Fatal pulmonary edema and pneumonitis after reexpansion of chronic pneumothorax. Chest 1971;60:399-401.

23. Lentz RJ, Lerner AD, Pannu JK. Routine monitoring with pleural manometry during therapeutic largevolume thoracentesis to prevent pleural-pressure-related complications: a multicentre, single-blind randomised controlled trial. Lancet Respir Med 2019;7:447-55.

24. Wan YY, Zhai CC, Lin XS, et al. Safety and complications of medical thoracoscopy in the management on pleural diseases. BMC Pulm Med 2019;19:125.

25. Clive AO, Jones HE, Bhatnagar R, et al. Interventions for the management of malignant pleural effusions: a network meta-analysis. Cochrane Database Syst Rev 2016;(5):CD010529.

26. Rahman NM, Pepperell J, Rehal S, et al. Effect of Opioids vs NSAIDs and Larger vs Smaller Chest Tube Size on Pain Control and Pleurodesis Efficacy Among Patients with Malignant Pleural Effusion: The TIME1 Randomized
Clinical Trial. JAMA 2015;314:2641-53.

27. Pathak V, Allender J, Grant M. Management of anticoagulant and antiplatelet therapy in patients undergoing interventional pulmonary procedures. Eur Respir Rev 2017;26:170020.

28. Puchalski JT, Argento AC, Murphy TE, et al. The safety of thoracentesis in patients with uncorrected bleeding risk. Ann Am Thorac Soc 2013;10:336-41.

29. Chen RB, Hu HJ, Matro E. Transcatheter arterial embolization for intercostal arterial bleeding in a patient after chest tube insertion. J Thorac Dis 2015;7:E194-7.

30. Chemelli AP, Thauerer M, Wiedermann F, et al. Transcatheter arterial embolization for the management of iatrogenic and blunt traumatic intercostal artery injuries. J Vasc Surg 2009;49:1505-13.

31. Tanizaki S, Maeda S, Sera M, et al. Small tube thoracostomy (20-22 Fr) in emergent management of chest trauma. Injury 2017;48:1884-7.

32. Inaba $K$, Lustenberger $T$, Recinos $G$, et al. Does size matter? A prospective analysis of 28-32 versus 36-40 French chest tube size in trauma. J Trauma Acute Care Surg 2012;72:422-7.

33. Kulvatunyou N, Joseph B, Friese R, et al. 14 French pigtail catheters placed by surgeons to drain blood on trauma patients: Is 14-Fr too small? J Trauma Acute Care Surg 2012;73:1423-7.

34. Fysh ETH, Tremblay A, Feller-Kopman D, et al. Clinical outcomes of indwelling pleural catheter-related pleural infections: an international multicenter study. Chest 2013;144:1597-602.

35. Lui MM, Thomas R, Lee YC. Complications of indwelling pleural catheter use and their management. BMJ Open Respir Res 2016;3:e000123.

36. Nie W, Liu Y, Ye J, et al. Efficacy of intrapleural instillation of fibrinolytics for treating pleural empyema and parapneumonic effusion: a meta-analysis of randomized control trials. Clin Respir J 2014;8;281-91.

37. Rahman NM, Maskell NA, West A, et al. Intrapleural use of tissue plasminogen activator and DNase in pleural infection. New Engl J Med 2011;365:518-26.

Cite this article as: Williams JG, Lerner AD. Managing complications of pleural procedures. J Thorac Dis 2021;13(8):52425250. doi: 10.21037/jtd-2019-ipicu-04 